\title{
Salafistische Netzwerke zwischen religiösem Wahrheitsanspruch, politischer Protestbewegung und Legitimierung von Gewalt
}

\author{
Ekkehard Rudolph
}

\section{Phänomenbeschreibung}

In den islamisch geprägten Ländern wie unter muslimischen Gemeinschaften westlicher Staaten hat das Glaubens- und Gesellschaftsmodell des Salafismus (arab. salafiya) in den letzten Jahren in beträchtlichem Maße an Einfluss gewonnen. Damit ist für die traditionellen islamischen Organisationen und Akteure eine neue dynamische Konkurrenz in der Auseinandersetzung um die Deutungshoheit über islamische Glaubenslehren entstanden.

Auch wenn die Zahlenstatistik ihnen lediglich eine marginale Rolle unter muslimischen Gemeinschaften zuerkennt, beschreiben Sicherheitsexperten die salafistischen Bewegungen insbesondere im Westen als eine der am schnellsten wachsenden Richtungen innerhalb des Islam. ${ }^{1}$

Dafür gibt es neben inneren und äußeren gesellschaftlichen Konfliktlinien, die der Bewegung Anhänger zutreiben, auch mediale Gründe. Denn die Bewegung versteht es, sich die neuen Medien effektiv zunutze zu machen. Als Plattformen zur Selbstdarstellung, Kommunikation und Vernetzung dienen Internet vor allem Web 2.0 - ebenso wie als reaktives Instrument zur Zurückweisung kritischer Mediendarstellungen. ${ }^{2}$

Zugleich haben sich aus der salafistischen Bewegung mittlerweile verschiedene, teilweise kontroverse Auslegungsrichtungen und Netzwerke entwickelt, die eine differenzierte Betrachtung verdienen.

\footnotetext{
${ }^{1}$ Vgl. das Statement der Leiterin des Berliner Verfassungsschutzes, in: Tagesspiegel (Berlin) vom 3.12.2008, unter http://www.tagesspiegel.de/politik/deutschland/Islamisten-Salafisten;art122,2675271 (05.12.2008); zur aktuellen Einschätzung des Salafismus vgl. Guido Steinberg 2012; Der Verfassungsschutzbericht für 2011 nennt die Zahl von 3.800 Personen, die dem Salafismus in Deutschland zugerechnet werden, abgerufen unter http://www.verfassungsschutz.de/de/publikationen/verfassungsschutz bericht/ (18.07.2012).

${ }^{2}$ Beispielhaft ist der Internetauftritt von „Islamically incorrect“ als Reaktion auf den rechtspopulistischen und islamkritischen Weblog „Politically incorrect“.
} 
Hintergrund für das Aufkommen salafistischer Strömungen ist der subjektiv empfundene Glaubens- und Werteverlust, den Teile der muslimischen Gemeinschaft als charakteristisch für die islamische Gemeinschaft (umma) insgesamt reklamieren. Von den geistigen Wortführern der Bewegung werden daraus Analogien zur historischen Vergangenheit des Islam und zu seinem universal gültigen Glaubens- und Rechtssystem konstruiert. Durch die vermeintliche Abweichung von der „wahren“ islamischen Lehre hätten sich die Muslime von ihren religiösen Ursprüngen entfernt und liefen Gefahr, ihre Glaubenswerte in einer mehrheitlich säkularen, nicht-islamischen Umgebung zu verlieren.

Als Leitbild dient der Bewegung das Beispiel der sogenannten „frommen Vorfahren" (al-salaf al-salih) oder der Prophetengefährten (sahaba), d.h. der Protagonisten der frühen muslimischen Gemeinde von Medina, die nach islamischer Lehre das „Zeitalter der Glückseligkeit“ (asr al-sa’ada), d. h. die Herrschaft des Islam in Abgrenzung von einer als religiös „unwissend“ ( jahil) wahrgenommenen Stammeskultur begründeten. Aus dem Ideal dieser heldenhaft verklärten Tradition bezieht der Salafismus seine Legitimation und Wirkungskraft bis heute.

Indem sie Geist und Buchstaben der prophetischen Tradition (Sunna) ohne Abstriche wiederzubeleben suchen und die vermeintlich regressiven gesellschaftlichen Entwicklungen der islamischen Welt - Salafisten bezeichnen diese als „unerlaubte Neuerungen“ (bid'a) - als Quelle aller politischen und sozialen Probleme betrachten, begeben sie sich in einen teilweise unauflösbaren (Identitäts-)Konflikt mit den Gesellschaftssystemen islamisch geprägter Länder wie den westlichen Demokratien.

Abweichend von den meisten islamistischen Bewegungen der Vergangenheit, die einen politischen Geltungsanspruch ihrer Ideologie und die Beeinflussung gesellschaftlicher Strukturen und Entwicklungen als extremistischen Gegenentwurf zu demokratischen Systemen offen vertreten, argumentieren viele salafistische Gelehrte und Prediger zunächst in einem religiös-theologischen Begriffsrahmen, der auf ein streng islamkonformes Regelverhalten des einzelnen Gläubigen zielt. In der Konsequenz allerdings hat das Islam-Verständnis salafistischer Akteure und deren buchstabengetreue Auslegung der offenbarten Texte eine Befürwortung frühislamischer Herrschafts- und Gesellschaftsformen zur 
Folge, die sich in einer mehr oder weniger deutlichen Antithese zu den Rechtsund Wertenormen der umgebenden Gesellschaften befindet. ${ }^{3}$

In religiöser Hinsicht ist das Bekenntnis zur Einheit und Einzigkeit Gottes (tauhid) für alle Salafisten von zentraler Bedeutung. In Fragen der Rechtsauslegung folgen sie fast ausschließlich der Schule (madhhab) der Hanbaliten, die sich in Fragen der religiösen Praxis und der sozialen Verhaltensregeln allein auf Aussagen in Koran und Sunna stützen und eine davon unabhängige Urteilsfindung in der islamischen Rechtslehre rigoros ablehnen. ${ }^{4}$

Der Kern salafistischer Lehre zeigt sich insbesondere in der Strömung des Wahhabismus (wahhabiyya), dessen Begründer der Religionsgelehrte Muhammad ibn Abd al-Wahhab (1703-1791) war und dessen Theorien sich in SaudiArabien als religiöse Staatsdoktrin nachhaltig ausgebreitet haben. ${ }^{5}$ So genießt das „Buch des Monotheismus“ (Kitab al-Tauhid) von Abd al-Wahhab, in dem die behauptete Abkoppelung des Islam von seinen religiösen Ursprüngen als Häresie gebrandmarkt wurde, in salafistischen Bewegungen bis heute Kultstatus. ${ }^{6}$

Gefördert durch die mediale Verbreitung religiöser Rechtsgutachten (fatwas), durch Publikationen und Finanzhilfen von Organisationen und Stiftungen, die in erster Linie der staatlichen Religionspolitik Saudi-Arabiens verpflichtet sind, finden salafistische Deutungen weite Verbreitung auch und gerade in der muslimischen Diaspora in Europa. Während die ältere Generation wahhabitischer Gelehrter eine vermeintlich politisch loyale, „puristische“ Form des Salafismus nach außen vertritt, haben Vertreter der jüngeren Generation - angeregt u. a. durch die Lage in Afghanistan seit den 1980er Jahren und das Bündnis des saudischen Königshauses mit den USA - eine militante Ausdrucksform des Salafismus theoretisch begründet und eingefordert. ${ }^{7}$

\footnotetext{
${ }^{3}$ Vgl. Bernard Haykel, On the Nature of Salafi Thought and Action, in: Roel Meijer 2009, S. 33 ff.; Marwan Abou-Taam 2012a.

${ }^{4}$ Die Rechtsschule geht auf den islamischen Theologen und Rechtsgelehrten Ahmad ibn Hanbal (gest. 855 in Bagdad) zurück. Vgl. Mathias Rohe 2011, S. 27 ff.

${ }^{5}$ Vgl. Werner Ende und Udo Steinbach 2005, S. $537 \mathrm{ff}$.

${ }^{6}$ Eine deutsche Übersetzung kursiert seit 2008 im Internet: Kitab at-Tawhid, übers. von Abu Izzudin, $1429 \mathrm{H}$.

${ }^{7}$ Vgl. Guido Steinberg 2005, S. 143 ff.
} 
In der Frage der offenen Legitimierung von Gewalt zur praktischen Durchsetzung islamischer Staatsvorstellungen ist die Bewegung bis heute gespalten. Während viele Salafisten den offensiven gewaltsamen Jihad (Ǧihäd, Dschihad) oder auch Selbstmordattentate als Verstoß gegen die Lehren des Islam ablehnen, wird die salafistische Bewegung seit den 1990er Jahren durch die Theorie zur Verteidigungspflicht der islamischen Länder gegen nichtislamische Besatzungsmächte und den Aufruf zum globalen Jihad massiv beeinflusst. Hier sind zahlreiche Schnittmengen zwischen den militanten Bewegungen und der „revolutionären" jihad-salafistischen Ideologie (Salafiya fihadiyya) erkennbar. ${ }^{8}$

In der Forschung werden salafistische Bewegungen deshalb seit Jahren in ihrer Divergenz zwischen verschiedenen Strömungen wahrgenommen, erstens einer apolitischen „puristischen“ Strömung, ${ }^{9}$ zweitens einer breiten missionarischen Strömung („Mainstream“- oder $D a$ 'wa-Salafismus) und drittens, einer Militanz einschließenden Strömung (Salafiya fihadiya), die sich dem Netzwerk Al-Qaida ideologisch angenähert hat. ${ }^{10}$ Im Zuge der dynamischen Entwicklung der salafistischen Denkschulen haben sich auch die Meinungsunterschiede und Kontroversen mit den politischen Bewegungen des Islamismus, vor allem mit der Muslimbruderschaft, deutlicher herausgebildet. ${ }^{11}$ Die theologische und rechtstheoretische Basis, auf die sich salafistische Strömungen zurückführen, weist jedoch - ungeachtet abweichender Auslegungen - viele Gemeinsamkeiten auf.

\section{Salafistische Ideologie im Internet}

Unter muslimischen Gemeinschaften in Deutschland findet die salafistische Ideologie in ihren verschiedenen Ausprägungen seit Jahren wachsende Anhängerzahlen. Eine Schlüsselrolle bei der Verbreitung spielt dabei das Medium Internet. Hier wird eine effektive Kommunikationsstrategie eingesetzt, die das Ziel

\footnotetext{
${ }^{8}$ Vgl. Thomas Hegghammer, Jihadi Salafis or Revolutionaries? In: Roel Meijer 2009, S. $244 \mathrm{ff}$.

${ }^{9}$ Die puristische, gewaltablehnende Variante des Salafismus wird vor allem von Gelehrten wie dem saudi-arabischen Scheich Rabi' al-Madkhali (geb. 1931) vertreten und unterhält auch in Europa Netzwerke von Anhängern.

${ }^{10}$ Vgl. Quintan Wiktorowicz 2006, S. $207 \mathrm{ff}$.

${ }^{11}$ Vgl. Olivier Roy 2006, S. $243 \mathrm{ff}$.
} 
der Verbreitung salafistischer Lehren im Sinne einer aktiven Ansprache von Zielgruppen mit den Bedürfnissen der modernen Mediengesellschaft verbindet.

So werden auf deutschsprachigen Internetseiten in nahezu unüberschaubarer Zahl Predigten und religiöse Abhandlungen auf Deutsch bzw. in deutscher Übersetzung bereitgestellt. Diese Entwicklung korrespondiert mit der weltweiten Zunahme salafistischer Webseiten mit Online-Predigten und „e-Fatwas“ vor allem saudisch-wahhabitischer Herkunft seit etwa 2000. ${ }^{12}$

In den letzten Jahren konnte die Mehrheit der Internetaufritte eher dem „Mainstream“-Salafismus zugeordnet werden, d. h., die Inhalte richteten sich auf die Vermittlung einer vermeintlich authentischen sunnitisch-islamischen Lehre und der darauf basierenden religiösen Orthopraxie in unterschiedlichen Alltagssituationen von Muslimen. Vor allem heterodoxe islamische Sekten und Bewegungen (Schiiten, Ahmadiyya u. a.), aber auch konkurrierende Islamauslegungen wurden ausgegrenzt.

Im Kern vermitteln die Betreiber vieler Internetseiten ein „geschlossenes“ islamisches Weltbild, das vor allem jungen Muslimen mit Migrationshintergrund und Konvertiten Immunität gegenüber Gewaltneigung auf der einen Seite wie gegen Identitätsverlust und "Verwestlichung“ auf der anderen Seite verspricht. Als Mittel dient ihnen das rigorose Einhalten islamischer Pflichten und Alltagsregeln, die teilweise bis in letzte Details vorgeschrieben werden. Einige Inhalte sind so fließend, dass die Grenzziehung zwischen einer rigoros-salafistischen Auslegung islamischer Überlieferungen und Intoleranz gegenüber anderen Auslegungen, die schließlich auch Sanktionen gegenüber „Abweichlern“ einschlieBen kann, nicht leicht zu ziehen ist.

Traktate und Predigten prominenter wahhabitischer Gelehrter bilden auf vielen salafistischen Internetseiten die Hauptautoritäten in der Auslegung islamischer Quellen. ${ }^{13}$ Typisch ist dabei die Ablehnung religiöser „Neuerungen“ und Abweichungen vom „wahren Islam“. Immanent wird die Wiederherstellung der

\footnotetext{
${ }^{12}$ Vgl. Dominique Thomas, Le role d'Internet dans la diffusion de la doctrine salafiste, in: Bernard Rougier 2008, S. 87 ff.; Der Ruf zu Gott 2011, S. 37 ff.

${ }^{13} \mathrm{Zu}$ den im „Mainstream"-Salafismus wie in Teilen des Jihad-Salafismus anerkannten Autoritäten gehören vor allem die Gelehrten Nasir al-Din al-Albani (1914-1999), Scheich Abd al-Aziz ibn Baz (gest. 1999) und Muhammad ibn al-Uthaimin (1926-2001).
} 
ursprünglichen Ordnung des Islam in den muslimischen Ländern als „Heilsweg“ propagiert.

Inhalte vieler salafistischer Webseiten zeigen eine Nähe zur Lehre des „Takfir", die eine zeitgemäße Auslegung des Koran und des islamischen Rechts als Beweise für „Unglauben“ ( $k u f r$ ) versteht und damit in Teilen eine religiöse Legitimation für deren Bekämpfung liefert. Auch wenn in der Konsequenz nicht zwingend eine Aufforderung zum aktiven und gewaltsamen Jihad propagiert wird, so ist feststellbar, dass hier eine kämpferische, an einer "dualistischen“ Welt- und Werteordnung orientierte Propaganda verbreitet wird, die dazu angelegt ist, Intoleranz und Desintegration unter muslimischen Gemeinschaften zu fördern. Hierdurch wird zum einen Intoleranz gegenüber „unislamischen“ Verhaltensweisen sowie gegenüber anderen Religionen und Glaubenslehren zum Ausdruck gebracht. Zum anderen wird die Orientierung auf das Weiterleben der „wahren Gläubigen“ im „Jenseits“ als vorrangige Glaubensnorm vertreten. Im Kern sind somit argumentative Brücken zur jihad-salafistischen Ideologie zu erkennen. ${ }^{14}$

\section{Multimediale Missionsbewegung}

In den letzten Jahren verbreiteten deutschsprachige Prediger, darunter zum Islam konvertierte Jugend-Imame wie Pierre Vogel, die Botschaften salafitischer Netzwerke mit ihrem multimedialen „Aufruf zum Islam“ (Da'wa) lokal und bundesweit über zahlreiche Vortrags- und Seminarveranstaltungen. ${ }^{15}$ Die Wochenendseminare zu islamischen Glaubensfragen, seit 2004 im Internet beworben und in Moscheegemeinden verschiedener deutscher Großstädte wie Berlin, Hamburg, Frankfurt, Köln und Bonn abgehalten, wurden durch eine Vielzahl von Vorträgen und Tagesveranstaltungen - teilweise auch als Open-Air-Event - zelebriert und um Prediger aus dem gesamten Bundesgebiet ergänzt. Die Teilnehmerzahlen zeigen den Sogeffekt der salafistischen $D a$ 'wa. Video-Mitschnitte von Vorträgen und Veranstaltungen werden über Internetportale wie YouTube, soziale Netzwerke oder über diverse Weblogs abrufbar gehalten. ${ }^{16}$

\footnotetext{
${ }^{14}$ Vgl. Marwan Abou-Taam 2012a.

${ }^{15}$ Die Zahl der Prediger wird aktuell auf ca. 50 geschätzt. Hinzu kommen jeweils einige Dutzend Anhänger, vgl. Guido Steinberg 2012, S. 6.

${ }^{16}$ In einem Internetforum wurden 2008 von einem Teilnehmer 30 maßgebliche deutschsprachige In-
} 
Viele Internetseiten popularisieren salafitisches Gedankengut im Sinne einer jugendnahen umfassenden islamischen Lebensordnung. Das Ziel, neue Anhänger zu werben und an die bestehenden Netzwerke zu binden, wird durch Vor-Ort-Veranstaltungen, Online-Unterweisungen via Paltalk und Möglichkeiten zum Download von Text-, Audio- und Videodateien deutlich. Auf einzelnen Webseiten nehmen religiöse „Schnellkurse“ und visualisierte Konversionen Jugendlicher zum Islam ebenso viel Raum ein wie Kritik an gesellschaftlichen Fragen und Problemen. Solche Themen finden auch in Internetforen Beachtung, in denen salafistische Deutungsangebote teilweise auch kontrovers diskutiert werden.

Schließlich sind auf vielen salafistischen Internetseiten auch kommerzielle Angebote einschließlich eines auf die Bedürfnisse der muslimischen Klientel angelegten Warenangebots zu finden. So können hier beispielsweise neben Büchern, Bild- und Tonträgern auch islamisch korrekte Kleidung und Ritualgegenstände online bestellt werden.

\section{Inhalte und Konsequenzen salafistischer Deutung}

Im Folgenden werden verschiedene salafistische Deutungsmuster vorgestellt. Auf salafistisch dominierten Webseiten und in Foren wird in der Konsequenz deutlich, wie extremistische Auslegungen religiöser Quellen eine intolerante, gesellschaftliche Desintegration fördernde und in Teilen gewaltlegitimierende Ideologie hervorbringen, mit der Protagonisten versuchen, die Deutungsmacht traditioneller muslimischer Akteure zu brechen.

\section{Pflicht zur Ablehnung des Taghut}

Auf salafistisch relevanten Internetseiten finden sich zahlreiche Texte über die Pflicht zur Ablehnung „nichtislamischer“ Rechtsordnungen. Ein Beispiel ist ein aus dem Arabischen übersetzter Vortrag unter dem Titel Die Ablehnung des Taghuts. Unter Zitierung des Koran, von Aussagen des Propheten (Hadîth) und

ternetseiten für „korrekte“ Informationen über den Islam angegeben. Inzwischen dürften FacebookAccounts und diverse Weblogs die Möglichkeiten der Verbreitung solcher Informationen um ein Vielfaches übersteigen. 
wahhabitischen Rechtsgelehrter wird davor gewarnt, „ungläubige“ Rechtsordnungen, Demokratie, Volksvertretungen etc. zu akzeptieren. Diese werden als Taghut (wörtlich: Götzen, d.h. dem Unglauben dienend) bezeichnet. Falls der Gläubige sich dennoch dem Taghut unterwerfe, falle er vom Islam ab, wobei Sanktionen für den „Abfall vom Islam“ nicht konkret benannt werden:

So ist dieser Säkularismus, der die Shari'a außer Kraft setzt, und das erlaubt, was Allah [...] verboten hat und das verbietet, was Allah [...] erlaubt hat, so muss man Kufr gegen ihn machen, denn er ist ein Taghut... ${ }^{17}$

In einem anderen Traktat zum gleichen Thema wird über demokratische Systeme Folgendes ausgesagt:

Die Demokratie ist ein Taghut und bildet das Fundament der Taghut, die außer Allah angebetet werden. Destotrotz zögern und scheuen sich die Menschen nicht, in diesen Din [d.h. Religion] einzutreten. Sie lassen sich vor ihr richten und loben ihn, ohne sich zu scheuen... ${ }^{18}$

\section{Abfall vom Islam}

Auf einer Internetseite werden die Folgen des Übertritts vom Islam unter islamrechtlichen Kriterien wie folgt wiedergegeben:

Die hanafitischen Ulama sind sich darüber einig, dass ein Mann, der sich vom Islam abgewendet hat (nachdem er Muslim war) getötet werden muss, eine Frau hingegen muss eingesperrt werden... ${ }^{19}$

Diese Rechtsauffassung wird u. a. in der Schrift Die Glaubenslehre der sunnitischen Gemeinschaft des in der salafistischen Bewegung als Autorität verehrten saudiarabischen Rechtsgelehrten Muhammad ibn al-Uthaimin (gest. 2001) verbreitet. Diese Haltung zur Sanktionierung der Apostasie gilt wegen ihrer Rückführung auf Hadîthe, nicht nur in der strengeren hanbalitischen Rechtsschule, als rechtlich unumstrittene Empfehlung. Ihre Durchsetzung sollte aller-

\footnotetext{
${ }^{17}$ Bishr ibn Fahd al-Bishr: Die Ablehnung des Taghut, Deutsche Übersetzung eines Vortragsreihe, erschienen im At-Taqwah Verlag, Riad, S. 42, abgerufen unter http://www.al-iman.net (02.06.2008, Seite ist nicht mehr im Netz).

${ }^{18}$ Die Erklärung des Begriffes Taghut, S. 22, abgerufen unter http://www.tawhed.de (02.04.2009).

${ }^{19}$ Vgl. Textauszug unter link „Die Aqida / Der Unterschied zwischen einem Murtadd (Abtrünnigen) und einem Kafir", abgerufen unter http://www.unserislam.de (02.06.2008).
} 
dings nach vorherrschender Meinung einem Rechtsgelehrten in einem islamischen Staatswesen vorbehalten bleiben. ${ }^{20}$

\section{Jenseitsorientierung}

In der salafistischen Deutung wird die Orientierung der Gläubigen auf den „Jüngsten Tag“ und das „Paradies“ besonders herausgehoben und mit der Sinnfrage und dem islamgemäßen Verhalten der Gläubigen verknüpft. Vorträge und Traktate beschreiben in oft suggestiv-emotionaler Art den Zustand der Gläubigen im Jenseits. ${ }^{21}$ Oft bestehen diese aus einer extrem kontrastierenden Beschreibung des Paradieses und im Gegensatz der Hölle als dem für die Gläubigen beziehungsweise Ungläubigen jeweils zu erwartendem Schicksal. Die teilweise drastische Beschreibung des "Jüngsten Gerichts“ (yaum al-qiyama) und der Selektion zwischen „Gläubigen“, „Ungläubigen“, „Heuchlern“ und „Sündern“ wird auf koranische und prophetische Überlieferungen zurückgeführt. Typisch ist die hier vermittelte Sicht auf das „Diesseits“, das als pauschal verkommen und wertlos erscheint und das dem Paradies als Ziel des „wahren Gläubigen“ gegenübergestellt wird. In einem Traktat aus dem Predigernetzwerk Die wahre Religion wird die Strafe im Sinne einer apokalyptischen Prophezeiung für diejenigen angedroht, die den Weg der „wahren Religion“ nicht beschreiten:

Diejenigen, die die Befehle Allahs in dieser Welt zurückweisen und, bewusst oder unbewusst, das Bestehen ihres Schöpfers leugnen, werden keine Rettung im Jenseits finden. Deshalb muss jeder, ohne Zeit zu verlieren, seine Situation begreifen und sich Allah stellen. Andernfalls wird er es bedauern und er sieht sich einem furchtbaren Ende gegenüber...22

\section{Abgrenzung von den „Ungläubigen“}

Mit dem aus dem Koran stammenden Begriffspaar al-wala wa al-bara - sinngemäß übersetzt mit „Loyalität zum islamischen Glauben und Lossagung vom

\footnotetext{
${ }^{20}$ Vgl. Silvia Tellenbach 2006.

${ }^{21}$ Vgl. dazu das im Internet verbreitete Traktat „Die Früchte der Investition“ von Abdullah $\mathrm{Mu}$ hammad Behdschat, das in deutscher Übersetzung vorliegt. Abruf unter http://www.way-to-allah.com (20.08.2008).

22 „Das Leben nach dem Tod“, in: Die Wahre Religion, Autor: nicht genannt, Hrsg. http://www.diewahre religion.de, S. 331.
} 
Unglauben“ - haben konservative islamische Rechtsgelehrte wie moderne islamistische Theoretiker ihre Rechtfertigung für das strikte Festhalten am Glauben und die Abgrenzung von den „Ungläubigen“ hergeleitet. ${ }^{23}$ Im Internet verbreitete salafistische Predigten und Traktate enthalten rigide Empfehlungen zum Verhalten von Muslimen gegenüber Ungläubigen und „abgefallenen“ Muslimen, die implizit auch deren Ausgrenzung und Bekämpfung einschließen. So findet sich in einer Abhandlung zu dem Thema folgendes Zitat:

Barah entspringt dem Hassen um der Religion willen... Hierzu gehört,... dass man Dschihad macht mit dem Geld, der Zunge und mit Waffen und dass man die Länder der Kuffar [d. h. der Ungläubigen] verlässt und zu den Ländern der Muslimin geht. . ${ }^{24}$

\section{Jihad-Rhetorik}

Bei der Forderung an die Muslime, das Glaubensbekenntnis und die strikte Einhaltung der Verhaltensregeln im Alltag durch die aktive Teilnahme am Jihad zu ergänzen, sind salafistische Bewegungen und Netzwerke bis heute tief gespalten. ${ }^{25}$ „Das Verhältnis zur Gewaltanwendung ist unter Salafisten entweder ambivalent, situationsbezogen oder eindeutig gewaltbejahend wie unter Jihadisten. ${ }^{\text {"26 }}$ Vor allem diskutiert wurde die Frage der islamrechtlichen Autorität, die den Jihad unter bestimmten Bedingungen ausrufen darf.

In einer Schrift aus dem in Saudi-Arabien angesiedelten „Islamic Propagation Office“ wird die Ambivalenz der Jihad-Deutung erkennbar:

Allah machte den Ğihad, den Kampf für Seine Sache, zu einer belohnten Handlung, an welche die Muslime glauben und welche sie praktizieren. Tatsächlich wird der Ğihad als der „Höcker“ des Kamels betrachtet, die höchste, wichtigste Stelle des Kamelkörpers, und genauso sollte dies für den Muslim gelten. Dem Kampf einzig und allein für

\footnotetext{
${ }^{23}$ Eine ausführliche Abhandlung zu der Lehre, die im Internet auch auf Deutsch angeboten wird, stammt von dem saudischen Gelehrten Muhammad Said al-Qahtani: Al-Wala wal-Bara, Loyalität und Lossagung für Allah nach der Aqida der Salaf.; Vgl. auch Joas Wagemakers, The Transformation of a Radical Concept - al-wala wa-l-bara in the Ideology of Abu Muhammad al-Maqdisi, in: Roel Meijer 2009, S. $81 \mathrm{ff}$.

${ }^{24}$ Traktat „Walah und Barah“, abgerufen unter http://www.al-iman.net (15.06.2008, aktuell nicht mehr im Netz)

${ }^{25}$ Vgl. Mariella Ourghi 2010, S. 26 ff.

${ }^{26}$ BMI 2011, S. 49.
} 
die Sache Allahs muss sich der ernsthafte gläubige Muslim in diesem Leben hingeben... ${ }^{27}$

Auf einer Internetseite sind detaillierte Ausführungen zum Umgang mit Nicht-Muslimen und „Abtrünnigen“, zum Jihad und zum islamischen Strafrecht enthalten, die sich auf den Umgang mit den gegnerischen Stammesverbänden zur Zeit des Propheten Mohammad in Medina beziehen, aber zugleich eine Handlungsanweisung für die Muslime in der Gegenwart enthalten:

Heutzutage, wo die imperialistischen Kuffar [d. h. Ungläubige] die islamischen Länder besetzt halten, ist der Djihad für jeden Gläubigen, der es vermag, eine 'ibadat im Range von Fard 'Ayn [d. h. unbedingte Glaubenspflicht $].{ }^{28}$

Diese Auslegung bietet, wie in vielen Vorträgen und Traktaten zum Thema Jihad, die im Internet verbreitet werden, Muslimen eine Legitimation für Gewalthandeln, insbesondere beim Verhalten in Krisengebieten unter „feindlicher“ Besatzung.

Der gewaltförmige Widerstand gegen Besatzungsmächte z.B. in Afghanistan wird von salafistischen Akteuren im Internet seit Jahren religiös gerechtfertigt. Dabei versuchen Verbreiter jihad-salafistischer Propaganda, die Legitimation mit religiösen Quellen, aber auch mit den Schriften der Vordenker militant-islamistischer Bewegungen zu begründen. Diese Schriften reichen von frühen Theoretikern wie Sayyid Qutb (Muslimbruderschaft, 1906-1966) bis zu den Ideologen des militanten Islamismus wie Abdallah Azzam (1941-1989) und Abu Muhammad al-Maqdisi (geb 1959). Deren ins Deutsche übersetzte Traktate können auch über relevante Internetforen heruntergeladen werden. So werden die zentralen Argumentationslinien der Takfir-bzw. Fihad-Ideologie an Interessierte vermittelt.

Die Quintessenz der Propaganda wurde vor einigen Jahren in einem deutlichen Aufruf an die Muslime zum Anschluss an den Jihad gegen alle „Ungläubigen" und ihre Verbündeten, darunter den Juden, mit folgenden Worten verbreitet:

Und wir rufen zum ernsthaften I'dād (Ausbildung und Vorbereitung) auf allen Ebenen und Arten (Rüstung, physisch, finanziell, mental und in Taqwā [d.h. Gottesfurcht])

\footnotetext{
${ }^{27}$ Abdul Rahman Al-Sheha: Missverständnisse über Menschenrechte im Islam. Riyadh 2003, S. 22.

${ }^{28}$ Textauszug, abgerufen unter http://www.unserislam.de (17.07.2008).
} 
für den Jihād auf dem Wege Allāhs und zur größtmöglichen Anstrengung in der fortwährenden Konfrontation gegen die Tawāghīt [d. h. Götzen] und ihrer Helfer - gegen die Yahūd [d.h. Juden] und ihre Verbündeten, um die Muslime und ihre Ländereien von den Fesseln der Demut und Besatzung zu befreien... ${ }^{29}$

\section{Entwicklung salafistischer Netzwerke in Deutschland}

Die salafistische Szene in der Bundesrepublik hat sich nach der Jahrtausendwende schrittweise etabliert. Vorläufer salafistisch inspirierter Zentren in den 90er Jahren waren das Multikulturhaus (MKH) in Neu-Ulm (Verbot 2005) und das Islamische Informationszentrum (IIZ) in Ulm (Verbot 2007). Hieraus gingen Akteure hervor, die sich ideologisch in Teilen der globalen jihad-salafistischen Bewegung verbunden fühlten, darunter Exil-Ägypter mit Kontakten zu salafistischen Netzwerken in Ägypten, Saudiarabien u. a. Die Grauzone zur Aktionsbereitschaft bei einzelnen Mitgliedern des Ulmer Milieus zeigte sich spätestens 2007 durch die Aufdeckung von Anschlagsplanungen der sogenannten „Sauerland-Gruppe“ ${ }^{30}$

Seit 2004 nahmen Islamseminare zur religiösen Unterweisung, die im Internet angekündigt wurden („Lerne den Islam“), zunächst in Bonn und in Berlin die Rolle von multiplikatorischen Schulungen bei der salafistischen Deutung islamischer Glaubenslehren ein. Die Laienprediger Abul-Hussain (Leipzig), Abu Jamal (Bonn) und Abdul Adhim (Berlin) fungierten hier als religiöse Führer und zugleich ideologische Wortführer einer salafistischen Bewegung, die sich an bestimmte Autoritäten bzw. Netzwerke und deren Lehrmeinungen insbesondere in Syrien, Marokko u. a. arabischen Ländern anlehnte. ${ }^{31}$ Im Kern wurden hier vor allem Jugendliche gemahnt, sich an einem strikt islamkonformen und Integration ablehnenden Glaubens- und Verhaltensmuster zu orientieren, ohne auf politische Einflussmöglichkeiten mittels Medienkampagnen völlig zu verzichten (Politischer oder „Mainstream“-Salafismus).

Mit der „Salafisierung“ im Umfeld von Moscheegemeinden, insbesondere in Stadtquartieren mit starken multiethnischen Bevölkerungsanteilen, wuchs auch

\footnotetext{
${ }^{29}$ Textauszug, abgerufen unter http://www.aazara.net (16.09.2008, Seite ist nicht mehr im Netz).

${ }^{30}$ Vgl. Souad Mekhennet u. a. 2006, S. 46 ff. sowie Annette Ramelsberger 2008.

${ }^{31}$ Vgl. Klaus Hummel 2009.
} 
die Anziehungskraft der Bewegung auf Jugendliche. Der 2000 zum Islam konvertierte Ex-Boxer Pierre Vogel („Abu Hamza“) fand seitdem durch eine spezifische, von Lokalkolorit geprägte Ansprache und einem religiös-moralischem Pathos Einfluss vor allem auf Jugendliche mit Migrationshintergrund und Konvertiten. ${ }^{32}$ Durch die Etablierung des Netzwerks „Die wahre Religion“ (DWR) ab 2005 einschließlich von Schulungen („Was ist Islam“, „Der Sinn des Lebens“) und einer begleitenden Internetpräsenz gelang es Vogel zusammen mit dem Kölner Laienprediger Ibrahim Abou-Nagie, eine überregionale mediengestützte Bewegung salafistischer $D a^{\prime} w a$ zu installieren, die vor allem die Konversion Jugendlicher zum Islam - gewissermaßen „im Schnellverfahren“ - forcierte. Mit einfachen Slogans gelang es verschiedenen Predigern, den Islam mehr und mehr als Antwort auf Sinn- und Identitätskrisen Jugendlicher zu empfehlen. ${ }^{33}$

Nach taktischen Meinungsverschiedenheiten unterbrach Vogel 2008 die Zusammenarbeit mit Abou-Nagie und etablierte in der Folgezeit das Netzwerk „Einladung zum Paradies“ (EZP), von dem in der Folgezeit Veranstaltungen mit „Event"-Charakter und internationalen Gastpredigern aus der salafistischen Szene ausgingen.

2009 erfolgte eine Verschmelzung mit den missionarischen Aktivitäten des türkischstämmigen Islampredigers Abu Anas, Initiator der „Online“-Islamschule in Braunschweig. Die Idee, die Islamschule, die nach dem Lehrverständnis wahhabitischer Gelehrsamkeit ausgerichtet ist, als Aus- und Fortbildungseinrichtung nach Mönchengladbach zu verlegen und mit der ortsansässigen Moschee zu verschmelzen, mündete ab 2010 in einer Kampagne um den Einfluss des salafistischen „Mainstreams“ auf Teile der muslimischen Gemeinschaft. Im Rahmen von lokalen juristischen Auseinandersetzungen, die von den öffentlichkeitswirksamen Aktivitäten einer Bürgerinitiative gegen Salafismus begleitet wurden, polarisierten Vogel und Vertreter der Bürgerinitiative auch vehement in den Medien. ${ }^{34}$ Nach dem Verzicht auf die Bauplanung und der Auflösung des Vereins „Einladung zum Paradies“ näherte sich Vogel seit 2011 wieder dem Netzwerk „Die wahre Religion“ um den salafistischen Prediger Ibrahim Abou-Nagie

\footnotetext{
${ }^{32}$ Vgl. Julia Gerlach, Die lässigen Gehirnwäscher, in: DIE ZEIT, Nr. 41, 2007.

${ }^{33}$ Vgl. Wolf Schmidt, Allah statt Playstation: Salafismus in Deutschland, in: Tageszeitung, 27. Juli 2009.

${ }^{34}$ Vgl. dazu Jörg Lau, Willkommen im Paradies, in: DIE ZEIT, Nr. 42, 2011.
} 
an, die laut einer aktuellen Analyse der „Stiftung Wissenschaft und Politik“ als „rechter Flügel des politischen Salafismus“ giltt. ${ }^{35}$ Aus dem Kölner Netzwerk heraus wurde 2011 schließlich auch die Kampagne der kostenlosen Koranverteilung in deutschen Großstädten („Lies!“) entwickelt, die nach Ansicht von Beobachtern die Bindewirkung zwischen diversen salafistischen Flügeln durch traditionelle $D a^{\prime}$ wa herzustellen sucht, in dem sie die religiöse Quelle des Islam neu ins Zentrum rückt. ${ }^{36}$

Die Problematik der Segregation und sukzessive ideologischen Radikalisierung innerhalb salafistischer Netzwerke kann aktuell durch die Etablierung der Plattform Millatu Ibrahim (dt. Religionsgemeinschaft Abrahams) seit Ende 2011 nachverfolgt werden. In ideologischer Anbindung an Akteure aus dem Netzwerk DWR gelang es einigen in Österreich und Deutschland agierenden Protagonisten (Abu Usama al-Gharib, Ebu Tejma, Abou Maleeq) durch Islamseminare und eine dicht gestaffelte Internetpräsenz in kurzer Zeit Anhänger für eine gewaltaffine salafistische Ideologie zu formieren. ${ }^{37}$ Deren ideologischer Resonanzboden lässt sich auf jihad-salafistische Akteure u. a. in Jordanien, Ägypten und Marokko zurückführen, die wie der einflussreiche Theoretiker des Jihad, Abu Muhammad al-Maqdisi, teilweise in ihren Ländern inhaftiert sind. Die Konfrontation der international vernetzten Plattform von Millatu Ibrahim mit der islamfeindlichen „Pro-Bewegung“, die mit provokativ enthüllten Muhammad-Karikaturen einen Landtags-Wahlkampf gegen die Präsenz des Islam in Deutschland insgesamt zu führen versuchte, mündete im Frühjahr 2012 in gewaltsamen Auseinandersetzungen in einigen Städten in Nordrhein-Westfalen. ${ }^{38}$

Auch wenn die medienwirksame Selbstvermarktung solcher Gruppierungen, die an die Jihad-Ideologie anknüpft und sie aktiv propagiert, deren realen Einfluss auf den salafistischen „Mainstream“ verschleiert, ist die Rasanz der Ent-

\footnotetext{
${ }^{35}$ Guido Steinberg 2012, S. 7.

${ }^{36}$ Vgl. Florian Flade, Ein Koran in jedem deutschen Haushalt, in: Die Welt vom 08.04.2012, abgerufen unter http://www.welt.de/politik/deutschland/article106163929/Ein-Koran-in-jedem-deutschen-Haushalt.html (10.04.2012).

${ }^{37}$ Vgl. Souad Mekhennet, Austrian Returns, Unrepentant, to Online Jihad. In: New York Times, 15.11.2011.

${ }^{38} \mathrm{Zu}$ den Auseinandersetzungen zwischen salafistischen Akteuren und Pro NRW vgl. den Kommentar von Heinrich Wefing, Liberal, aber nicht blöd, in: DIE ZEIT vom 10.05.2012.
} 
wicklung und Polarisierung innerhalb der salafistischen $D a$ 'wa-Bewegung, die auch in anderen europäischen Ländern zu beobachten ist, bemerkenswert. Nicht zuletzt stellt sie auch die Integrations- und Sicherheitspolitik vor neue Herausforderungen. ${ }^{39}$

Rückblickend ist die rasante Entwicklung vor allem informeller salafistischer Strukturen - hierin unterscheiden sich Akteure und Anhängerschaft von formal und hierarchisch strukturierten islamischen Organisationen wie Milli Görüs (= „Nationale Sicht“ - Islamische Gemeinschaft Milli Görüs) auch eine Entwicklung hin zu einer wachsenden Binnendifferenzierung. So sind zwischen den verschiedenen Strömungen im Internet starke Rivalitäten und Deutungskontroversen zu beobachten, die eine organisatorische Vereinigung in einer einheitlichen Struktur in Zukunft nicht realistisch erscheinen lassen. ${ }^{40}$

\section{Mehrheitsislam und Salafismus}

Auf die Herausforderung salafistischer Netzwerke hinsichtlich ihrer Einflussnahme auf gesellschaftliche Entwicklungen, insbesondere auf Integration und Teilhabe von Muslimen in Alltag und Politik, ist in der Bundesrepublik wie in anderen europäischen Nachbarländern, die sich mit dem Phänomen auseinandersetzen, noch keine erfolgreich erprobte Präventionsstrategie erkennbar. ${ }^{41}$ Gleichwohl sind in den letzten Jahren zahlreiche Projekte initiiert und damit Bemühungen verstärkt worden, einer Radikalisierung durch salafistische Ideologie insbesondere unter Jugendlichen auf unterschiedlichen Ebenen vorzubeugen. Diese richten sich vor allem an Pädagogik, Jugend- und Sozialarbeit, Familien und Politik. ${ }^{42}$

Die kritische Auseinandersetzung mit extremistischen Auslegungen islamischer Glaubenslehren ist aber auch Aufgabe islamischer Gemeinschaften und

\footnotetext{
${ }^{39}$ Vgl. dazu F. W. Horst 2012; Albrecht Metzger 2012.

${ }^{40}$ Vgl. BMI 2011, S. 11.

${ }^{41}$ Vgl. u. a. National Coordinator for Counterterrorism (Netherlands) 2008.

${ }^{42}$ Vgl. Konrad-Adenauer-Stiftung 2011; Claudia Dantschke 2011, S. $78 \mathrm{ff}$. Erfolgversprechend ist auch das Projekt „Islam, Islamismus \& Demokratie - Filme für die pädagogische Arbeit mit jungen Muslimen“ der Hochschule für Angewandte Wissenschaften in Hamburg, vgl. dazu http://www. haw-hamburg.de/index.php?id = 25915\&type $=98$ (30.07.2012).
} 
Organisationen, deren Selbstverständnis als religiöse Minderheit und als Bürger in einem demokratischen Verfassungsstaat in Frage gestellt wird.

Dieser Aufgabe der Prävention nach innen nehmen muslimische Dachverbände nicht immer nach außen transparent wahr, sodass gelegentlich der Eindruck entsteht, dass die notwendige Bekämpfung xenophober oder islamfeindlicher Tendenzen und ihrer Protagonisten in der Gesellschaft eine weit wichtigere Rolle in der Außendarstellung spielt als die offene Auseinandersetzung mit sich ausweitenden extremistischen und desintegrativen Ideologien unter muslimischen Gemeinschaften.

Umso bemerkenswerter ist es, wenn in einem Kommentar eines muslimischen Journalisten dazu selbstkritisch wie folgt Stellung genommen wird:

Es zählt zu den Schwachstellen des organisierten Islam in Deutschland, dass sich dieser in den letzten zehn Jahren zwar oft - und kompromisslos wie eindeutig - gegen „Terror" und „Extremismus" aussprach. Die Ideologie und falsche Lesart des Islam aber, die deren Grundlage bildete, blieb mehrheitlich ausgespart. Vielleicht weil es den Verbänden an geeigneten und mutigen Gelehrten fehlt, vielleicht, weil die Vorstellung, wir seien „doch alles Brüder“, bislang dominierte; bisher jedenfalls fehlt eine fundierte, rechtlich überzeugende Zurückweisung dieser Ideologie. ${ }^{43}$

Jüngste Äußerungen und Kommentare belegen, dass Vertreter der muslimischen Dachverbände in der Bundesrepublik die Herausforderung, dass vor allem junge Muslime in wachsendem Umfang in der Gefahr stehen, sich von selbsternannten salafistischen Predigern in eine „moderne Irrlehre“ hineinziehen zu lassen, erkannt haben. ${ }^{44}$ Gleichwohl sind Bemühungen, die praktische Immunität der überwältigenden Mehrheit der Muslime in Deutschland gegenüber „Radikalismen" nachhaltig in den Vordergrund zu rücken, für die öffentliche Wahrnehmung des Islam und der muslimischen Akteure von erheblicher Bedeutung.

\footnotetext{
${ }^{43}$ Sulaiman Wilms, Hilfreiche Radikale, in: Islamische Zeitung vom 28.06.2011, abgerufen unter http://www.islamische-zeitung.de/:id=14843 (07.07.2011).

${ }^{44}$ Vgl. Khalil Breuer, Rational und differenziert, in: Islamische Zeitung vom 14.06.2012, abgerufen unter http://www.islamische-zeitung.de/:id=15840 (15.06.2012); Interview „Extremismus können wir nur gemeinsam bekämpfen" mit Aiman Mazyek, Vorsitzender des Zentralrats der Muslime (ZMD), unter http://www.tagesschau.de/inland/integration212.html (05.03.2012); vgl. auch Kommentar ,Jenseits von Eden“ von Muhammad Murtaza zu den Anschlägen von Toulouse auf der Webseite des ZMD, eingestellt am 22.03.2012, unter http://www.islam.de/20027.php (Datum: 25.03.2012); vgl. auch Muhammad Sameer Murtaza 2012.
} 


\section{Fazit}

Vor allem das Internet trägt zum schnellen und passgenauen Transfer von salafistischer Ideologie bei. Dadurch sind neue informelle Gemeinschaften mit überregionalen islamischen Autoritäten wie Laienpredigern oder Jugendimamen entstanden, die häufig in internationale salafistische Netzwerke eingebunden sind.

Trotz theoretischer Abgrenzungen zwischen Verfechtern einer „wahren Religion“, den Akteuren politischer Protestbewegungen beispielsweise gegen Erscheinungsformen von Islamophobie und den Verfechtern einer aktiven JihadIdeologie bestehen häufig enge Verlinkungen in Doktrin und Rechtsauffassung, deren differenziertes Verständnis Wissenschaft, Medien und Politik vor komplexe Herausforderungen stellt. Auch wenn zu differenzieren ist, inwieweit salafistische Überzeugungen allein Gewaltbereitschaft hervorbringen oder fördern, ist festzustellen, dass eine militant-islamistische Ideologie ihre Legitimation nahezu ausschließlich aus dem Reservoir salafistischer Deutungen zieht. Daraus folgt, dass die Art und Weise der Beeinflussung beachtlicher Teile, überwiegend, aber nicht ausschließlich muslimischer Jugendlicher durch salafistische Akteure sowohl auf die Tagesordnung muslimischer Verbände wie der Integrations- und Sicherheitsbehörden gehört. Zudem erscheint es unverzichtbar, dass im islamischen Religionsunterricht das Verhältnis von Selbstbestimmung, gesellschaftlicher Verantwortung und religiöser Orientierung im Zentrum steht.

Während strafrechtliche und verfassungsschutzrelevante Sachverhalte von den Sicherheitsbehörden bearbeitet werden, entziehen sich die vielfältigen Formen radikalisierender Einflussnahme durch verzerrende Propaganda häufig der flächendeckenden Beobachtung und Bewertung durch gesellschaftliche Verantwortungsträger. Im Rahmen wissenschaftlicher Analysen ist deshalb anzuraten, die „Trichterfunktion“ etwa des Salafismus für Radikalisierungs- und Desintegrationsprozesse nicht aus den Augen zu verlieren. Schließlich sollte der vor allem über das Internet gesteuerte Ideologietransfer durch lokale, nationale und internationale Netzwerke mit den Möglichkeiten „dekonstruierender“ Medien beeinflusst werden, um vor allem jugendliche Zielgruppen zu befähigen, einseitige Verführungsversuche durch eigene Erkenntnisse und Wahrnehmungen zu relativieren. Hier liegt ein Förderungspotential, das besonders für lokale und 
überregionale Präventionsprojekte sowie die Sozial- und Schulpädagogik mit Jugendlichen, die im Kontakt zu salafistischen Milieus stehen, hilfreich und notwendig ist. ${ }^{45}$

\section{Literatur}

Dantschke, Claudia, Ahmad Mansour u. a.: „Ich lebe nur für Allah“ - Argumente und Anziehungskraft des Salafismus, in: Handreichung für Pädagogik, Jugend- und Sozialarbeit, Familien und Politik. Schriftenreihe Zentrum für Demokratische Kultur, Berlin 2011.

Ende, Werner und Steinbach, Udo (Hrsg.), Der Islam in der Gegenwart. 5. Auflage. München 2005.

Horst, F.W., Gewalttätige Gegenkultur, in: Frankfurter Allgemeine Zeitung vom 10.05.2012.

Hummel, Klaus, Salafismus in Deutschland - eine Gefahrenperspektive. Stand: Juni 2009. Analysepapier (unveröffentlicht). LKA Sachsen.

Konrad-Adenauer-Stiftung (Hrsg.), Islamismus!? Die wichtigsten Fragen und Antworten zu religiöser Radikalisierung bei Jugendlichen - Eine Handreichung für Pädagoginnen und Pädagogen, Sankt Augustin 2011.

Meijer, Roel (Hrsg.), Global Salafism: Islam's New Religious Movement, London/New York 2009.

Mekhennet, Souad, Sautter, Claudia, Hanfeld, Michael, Die Kinder des Dschihad: Die neue Generation des islamitischen Terrors in Europa, München 2006.

Murtaza, Muhammad Sameer, Mohammeds Erben: Was ist Salafismus - und warum wurde er zu einer gewalttätigen Ideologie? In: DIE ZEIT, Nr. 25 vom 21.6.2012.

Ourghi, Mariella, Muslimische Positionen zur Berechtigung von Gewalt - Einzelstimmen, Revisionen, Kontroversen, Würzburg 2010.

Ramelsberger, Annette, Der deutsche Dschihad. Berlin 2008.

Rohe, Mathias, Das islamische Recht: Geschichte und Gegenwart, München (3. Aufl.) 2011.

Rougier, Bernard (Hrsg.), Qu'est-ce-que le salafisme? Paris 2008.

Roy, Olivier, Der islamische Weg nach Westen - Globalisierung, Entwurzelung und Radikalisierung, München 2006.

Steinberg, Guido, Der nahe und der ferne Feind - Die Netzwerke des islamistischen Terrorismus, München 2005.

${ }^{45}$ Vgl. aktuelle Projekte des Berliner ZDK (http://www.zentrum-fÃijr-demokratische-kultur.de), der Aktion Courage (http://www.schule-ohne-rassismus.org) oder des Vereins „ufuq e. V.“ (http://www.ufuq.de), die in den letzten Jahren als zivilgesellschaftliche Träger von beispielhaften Präventionsinitiativen hervorgetreten sind. 
Wiktorowicz, Quintan, Anatomy of the Salafi Movement. In: Studies in Conflict \& Terrorism, Philadelphia. Nr. 29, 2006, S. 207-239.

\section{Internetquellen}

Abou-Taam, Marwan (2012a), Die Salafiyya - eine kritische Betrachtung, in: Bundeszentrale für Politische Bildung (Hrsg.), Bonn 2012, unter http://www.bpb.de/politik/ extremismus/islamismus/138468/die-salafiyya-eine-kritische-betrachtung (25.07.2012).

Abou-Taam, Marwan (2012b), Die Salafiyya-Bewegung in Deutschland, in: Bundeszentrale für Politische Bildung (Hrsg.), Bonn 2012, unter http://www.bpb.de/politik/extremis mus/islamismus/136705/die-salafiyya-bewegung-in-deutschland (25.07.2012).

BMI - Bundesministerium des Innern (Hrsg.), Lagebild zur Verfassungsfeindlichkeit salafistischer Bestrebungen, vorgelegt der Innenministerkonferenz am 22.06.2011, unter http://www.bundesrat.de/cln_117/DE/gremien-konf/fachministerkonf/imk/Sitzungen/ 11-06-22/anlage14, templateld $=$ raw, property $=$ publicationFile.pdf/anlage14.pdf $\quad(30.07$. 2011).

Bundesamt für Verfassungsschutz (Hrsg.), Salafistische Bestrebungen in Deutschland. Köln 2012, unter: http://www.verfassungsschutz.de/de/publikationen/Islamismus/brosch uere_1204_salafistische_bestrebungen/ (30.04.2012).

Metzger, Albrecht, Viel Populismus, wenig strategisches Denken: Die Salafisten und die deutsche Sicherheitspolitik, in: Internetportal „Qantara“ vom 11.07.2012, unter http: //de.qantara.de/Viel-Populismus-wenig-strategisches-Denken/19470c20666i0p8/index. html (15.07.2012).

Ministerium für Inneres und Kommunales des Landes Nordrhein-Westfalen, Abteilung Verfassungsschutz (Hrsg.), Der Ruf zu Gott - Formen salafistischer Propaganda, Düsseldorf 2011, unter http://www.mik.nrw.de/verfassungsschutz/islamismus.html (30.11.2011).

National Coordinator for Counterterrorism Netherlands (Hrsg.), Salafism in the Netherlands: A passing phenomenon or a persistent factor of significance? 2008. Abrufbar unter: http://www.nctb.nl (24.07.2008).

Steinberg, Guido, Wer sind die Salafisten - Zum Umgang mit einer schnell wachsenden und sich politisierenden Bewegung. Berlin: Stiftung Wissenschaft und Politik, SWPAktuell Nr. 28, Mai 2012, unter http://www.swp-berlin.org/fileadmin/contents/products/ aktuell/2012A28_sbg.pdf (25.05.2012).

Tellenbach, Silvia, Die Apostasie im islamischen Recht, in: Gesellschaft für Arabisches und Islamisches Recht - GAIR - (Hrsg.), Stand: 2006, unter http://www.gair.de/pdf/ publikationen/tellenbach_apostasie.pdf (30.7.2008). 
